\title{
The Energy and Operations of Graphs
}

\author{
Haicheng Ma, Xiaohua Liu \\ Department of Mathematics, Qinghai Nationalities University, Xining, China \\ Email: qhmymhc@163.com
}

How to cite this paper: Ma, H.C. and Liu, X.H. (2017) The Energy and Operations of Graphs. Advances in Pure Mathematics, 7, 345-351.

https://doi.org/10.4236/apm.2017.76021

Received: April 25, 2017

Accepted: June 18, 2017

Published: June 21, 2017

Copyright (c) 2017 by authors and Scientific Research Publishing Inc. This work is licensed under the Creative Commons Attribution International License (CC BY 4.0).

http://creativecommons.org/licenses/by/4.0/

\begin{abstract}
Let $G$ be a finite and undirected simple graph on $n$ vertices, $A(G)$ is the adjacency matrix of $G, \lambda_{1}, \lambda_{2}, \cdots, \lambda_{n}$ are eigenvalues of $A(G)$, then the energy of $G$ is $\varepsilon(\mathbb{G})=\sum_{i=1}^{n}\left|\lambda_{i}\right|$. In this paper, we determine the energy of graphs obtained from a graph by other unary operations, or graphs obtained from two graphs by other binary operations. In terms of binary operation, we prove that the energy of product graphs $G_{1} \times G_{2}$ is equal to the product of the energy of graphs $G_{1}$ and $G_{2}$, and give the computational formulas of the energy of Corona graph $G \circ H$, join graph $G \nabla H$ of two regular graphs $G$ and $H$, respectively. In terms of unary operation, we give the computational formulas of the energy of the duplication graph $D_{m} G$, the line graph $L(G)$, the subdivision graph $S(G)$, and the total graph $T(G)$ of a regular graph $G$, respectively. In particularly, we obtained a lot of graphs pair of equienergetic.
\end{abstract}

\section{Keywords}

Graph, Matrix, Energy, Operation

\section{Introduction}

Let $G$ be a finite and undirected simple graph, with vertex set $V(G)$ and edge set $E(G)$. The number of vertices of $G$ is $n$, and its vertices are labeled by $v_{1}, v_{2}, \cdots, v_{n}$. The adjacency matrix $A(G)$ of the graph $G$ is a square matrix of order $n$, whose $(i, j)$-entry is equal to 1 if the vertices $v_{i}$ and $v_{j}$ are adjacent and is equal to zero otherwise. The characteristic polynomial of the adjacency matrix, i.e., $\operatorname{det}\left(x I_{n}-A(G)\right)$, where $I_{n}$ is the unit matrix of order $n$, is said to be the characteristic polynomial of the graph $G$ and will be denoted by $\phi(G, x)$. The eigenvalues of a graph $G$ are defined as the eigenvalues of its adjacency matrix $A(G)$, and so they are just the roots of the equation $\phi(G, x)=0$. since $A(G)$ is a real symmetric matrix, so its eigenvalues are all real. Denoting them 
by $\lambda_{1}, \lambda_{2}, \cdots, \lambda_{n}$ and as a whole, they are called the spectrum of $G$. Spectral properties of graphs, including properties of the characteristic polynomial, have been extensively studied, for details, we refer to [1]. In the 1970s, I. Gutman in [2] introduced the concept of the energy of $G$ by

$$
\varepsilon(G)=\sum_{i=1}^{n}\left|\lambda_{i}\right|
$$

In the Hückel molecular orbital (HMO) theory, the energy approximates the the molecular orbital energy levels of $\pi$-electrons in conjugated hydrocarbons (see [3] [4] [5] [6]). Up to now, the energy of $G$ has been extensively studied, for details, we refer to [7] [8] [9]. In this paper, we determine the energy of graphs obtained from a graph by other unary operations, or graphs obtained from two graphs by other binary operations. In terms of binary operation, we prove that the energy of product graphs $G_{1} \times G_{2}$ is equal to the product of the energy of graphs $G_{1}$ and $G_{2}$, and give the computational formulas of the energy of Corona graph $G \circ H$, join graph $G \nabla H$ of two regular graphs $G$ and $H$, respectively. In terms of unary operation, we give the computational formulas of the energy of the duplication graph $D_{m} G$, the line graph $L(G)$, the subdivision graph $S(G)$, and the total graph $T(G)$ of a regular graph $G$, respectively. In particularly, we obtained a lot of graphs pair of equienergetic.

Two nonisomorphic graphs are said to be equienergetic if they have the same energy. Let $G$ and $H$ be two vertex disjoint graphs, $G \cup H$ denotes the union graph of $G$ and $H . m G$ denoted the union graph of $m$ copies of $G$. $K_{n}$ denotes the complete graph with $n$ vertices. For more notation and terminology, we refer the readers to standard textbooks [10].

\section{The Binary Operations of Graphs}

Let $G_{1}$ and $G_{2}$ be two graphs with vertex set $V\left(G_{1}\right)$ and $V\left(G_{2}\right)$ respectively. the product $G_{1} \times G_{2}$ is the graph with vertex set $V\left(G_{1}\right) \times V\left(G_{2}\right)$, in which two vertices, say $\left(x_{1}, y_{1}\right)$ and $\left(x_{2}, y_{2}\right)$, are adjacent if and only if $x_{1}$ is adjacent to $x_{2}$ in $G_{1}$ and $y_{1}$ is adjacent to $y_{2}$ in $G_{2}$. Let $A=\left(a_{i j}\right)_{m \times n}$, $B=\left(b_{i j}\right)_{p \times q}$ be two matrices, the Kronecker product $A \otimes B$ of $A$ and $B$ is the $m p \times n q$ matrix obtained from $A$ by replacing each element $a_{i j}$ with the block $a_{i j} B$.

Lemma 2.1. [1] Let $A\left(G_{1}\right), A\left(G_{2}\right)$ be adjacency matrices of graphs $G_{1}$, $G_{2}$, respectively. Then the product graph $G_{1} \times G_{2}$ has as adjacency matrix $A\left(G_{1}\right) \otimes A\left(G_{2}\right)$.

Lemma 2.2. [11] Let $A, B, C, D$ be matrices and the products $A C, B D$ exist. Then

$$
(A \otimes B)(C \otimes D)=(A C) \otimes(B D) .
$$

Theorem 2.1. Let $G, H$ be two graphs. Then

$$
\varepsilon(G \times H)=\varepsilon(G) \times \varepsilon(H) .
$$

Proof. Let $\lambda_{1}, \lambda_{2}, \cdots, \lambda_{n}$ and $\mu_{1}, \mu_{2}, \cdots, \mu_{m}$ be the eigenvalues of $G$ and $H$, respectively, suppose $x_{i}(i=1,2, \cdots, n)$ are linearly independent eigenvectors of 
$A(G)$ corresponding to $\lambda_{1}, \lambda_{2}, \cdots, \lambda_{n}$ respectively, and $y_{i}(i=1,2, \cdots, m)$ are linearly independent eigenvectors of $A(H)$ corresponding to $\mu_{1}, \mu_{2}, \cdots, \mu_{m}$ respectively, Consider the vector $z_{i j}=x_{i} \otimes y_{j}(i=1,2, \cdots, n, j=1,2, \cdots, m)$.

Using Lemma 2.1, we see that

$$
(A(G) \otimes A(H)) z_{i j}=\left(A(G) x_{i}\right) \otimes\left(A(H) y_{j}\right)=\lambda_{i} \mu_{j} x_{i} \otimes y_{j}=\lambda_{i} \mu_{j} z_{i j} .
$$

In this way, we find $m n$ linearly independent eigenvectors, and hence $\lambda_{i} \mu_{j}(i=1,2, \cdots, n, j=1,2, \cdots, m)$ are the eigenvalues of $G \times H$.

And so

$$
\varepsilon(G \times H)=\sum_{i=1}^{n} \sum_{j=1}^{m}\left|\lambda_{i} \mu_{j}\right|=\sum_{i=1}^{n}\left|\lambda_{i}\right| \sum_{j=1}^{m}\left|\mu_{j}\right|=\varepsilon(G) \varepsilon(H) .
$$

Corollary 2.1. Let $G_{1}, G_{2}, \cdots, G_{k}$ be $k$ graphs. Then

$$
\varepsilon\left(G_{1} \times G_{2} \times \cdots \times G_{k}\right)=\varepsilon\left(G_{1}\right) \varepsilon\left(G_{2}\right) \cdots \varepsilon\left(G_{k}\right) .
$$

Let $G$ be a graph with $n$ vertices, and let $H$ be a graph with $m$ vertices. The Corona $G \circ H$ is the graph with $n+m n$ vertices obtained from $G$ and $n$ copies of $H$ by joining the $i$-th vertex of $G$ to each vertex in $i$-th copy of $H(i=1,2, \cdots, n)$.

Lemma 2.3. [1] Let $G$ be a graph with $n$ vertices, and let $H$ be an $r$-regular graph with $m$ vertices. Then the characteristic polynomial of the corona $G \circ H$ is given by

$$
\phi(G \circ H, x)=\phi\left(G, x-\frac{m}{x-r}\right)(\phi(H, x))^{n} .
$$

Theorem 2.2. Let $G$ be a graph with $n$ vertices, and let $H$ be an $r$-regular graph with $m$ vertices. If $\lambda_{1}, \lambda_{2}, \cdots, \lambda_{n}$ and $r, \mu_{2}, \cdots, \mu_{m}$ be the eigenvalues of $G$ and $H$, respectively. then

$$
\varepsilon(G \circ H)=\frac{1}{2} \sum_{i=1}^{n}\left(\left|r+\lambda_{i}+\sqrt{\left(r-\lambda_{i}\right)^{2}+4 m}\right|+\left|r+\lambda_{i}-\sqrt{\left(r-\lambda_{i}\right)^{2}+4 m}\right|\right)+n(\varepsilon(H)-r) .
$$

Proof. By Lemma 2.3, we have

$$
\begin{aligned}
\phi(G \circ H, x) & =(x-r)^{n}\left(x-\mu_{2}\right)^{n} \cdots\left(x-\mu_{m}\right)^{n} \prod_{i=1}^{n}\left(x-\frac{m}{x-r}-\lambda_{i}\right) \\
& =\left(x-\mu_{2}\right)^{n} \cdots\left(x-\mu_{m}\right)^{n} \prod_{i=1}^{n}\left(x^{2}-\left(r+\lambda_{i}\right) x+r \lambda_{i}-m\right) .
\end{aligned}
$$

And so

$$
\begin{aligned}
\varepsilon(G \circ H) & =\frac{1}{2} \sum_{i=1}^{n}\left(\left|r+\lambda_{i}+\sqrt{\left(r-\lambda_{i}\right)^{2}+4 m}\right|+\left|r+\lambda_{i}-\sqrt{\left(r-\lambda_{i}\right)^{2}+4 m}\right|\right)+n\left(\sum_{j=2}^{m}\left|\mu_{j}\right|\right) \\
& =\frac{1}{2} \sum_{i=1}^{n}\left(\left|r+\lambda_{i}+\sqrt{\left(r-\lambda_{i}\right)^{2}+4 m}\right|+\left|r+\lambda_{i}-\sqrt{\left(r-\lambda_{i}\right)^{2}+4 m}\right|\right)+n(\varepsilon(H)-r) .
\end{aligned}
$$

Corollary 2.2. Let $H_{1}$ and $H_{2}$ be two equienergetic $r$-regular graph with $m$ vertices, and let $G$ be a graph with $n$ vertices. Then $G \circ H_{1}$ and $G \circ H_{2}$ are equienergetic. 
Corollary 2.3. Let $m \geq 2, n \geq 3$. Then

$\varepsilon\left(K_{n} \circ K_{m}\right)=m n+m-2+(n-1) \sqrt{m^{2}+4 m}$.

Proof. $K_{m}$ has spectrum $n-1,-1$ ( $n-1$ times). Since $(m-1)-1-\sqrt{(m-1+1)^{2}+4 m} \leq 0$, and $m \geq 2, n \geq 3$ means $(m-1)+(n-1)-\sqrt{(m-n)^{2}+4 m} \geq 0$. Hence

$$
\begin{aligned}
\varepsilon\left(K_{n} \circ K_{m}\right)= & \frac{1}{2} \sum_{i=1}^{n}\left(\left|(m-1)+\lambda_{i}+\sqrt{\left(m-1-\lambda_{i}\right)^{2}+4 m}\right|\right. \\
& \left.+\left|m-1+\lambda_{i}-\sqrt{\left(m-1-\lambda_{i}\right)^{2}+4 m}\right|\right)+n(\varepsilon(H)-(m-1)) \\
= & m+n-2+(n-1) \sqrt{m^{2}+4 m}+n(m-1) \\
= & m n+m-2+(n-1) \sqrt{m^{2}+4 m} .
\end{aligned}
$$

Let $G$ and $H$ be two graphs, The join $G \nabla H$ of (disjoint) grapgs $G$ and $H$ is the graph obtained from $G \cup H$ by joining each vertex of $G$ to each vertex of $H$.

Lemma 2.4. [1] If $G_{1}$ is $r_{1}$-regular with $n_{1}$ vertices, and $G_{2}$ is $r_{2}$ regular with $n_{2}$ vertices, then the characteristic polynomial of the join $G_{1} \nabla G_{2}$ is given by

$$
\phi\left(G_{1} \nabla G_{2}, x\right)=\frac{\phi\left(G_{1}, x\right) \phi\left(G_{2}, x\right)}{\left(x-r_{1}\right)\left(x-r_{2}\right)}\left(\left(x-r_{1}\right)\left(x-r_{2}\right)-n_{1} n_{2}\right) .
$$

Corollary 2.4. Let $G_{i}$ be $r_{i}$-regular graph with $n_{i}$ vertices, $i=1$,2. Then

$$
\varepsilon\left(G_{1} \nabla G_{2}\right)=\varepsilon\left(G_{1}\right)+\varepsilon\left(G_{2}\right)-\left(r_{1}+r_{2}\right)+\sqrt{\left(r_{1}+r_{2}\right)^{2}+4\left(n_{1} n_{2}-r_{1} r_{2}\right)} .
$$

Corollary 2.5. Let $G_{1}$ and $H_{1}$ be two equienergetic $r_{1}$-regular graph with $n_{1}$ vertices, and let $G_{2}$ and $H_{2}$ be two equienergetic $r_{2}$-regular graph with $n_{2}$ vertices, then $G_{1} \nabla G_{2}$ and $H_{1} \nabla H_{2}$ are equienergetic.

Lemma 2.5. [1] Let $G_{1}, G_{2}, \cdots, G_{k}$ be regular graphs, let $G_{i}$ have degree $r_{i}$ and $n_{i}$ vertices $(i=1,2, \cdots, k)$. where the relations $n_{1}-r_{1}=n_{2}-r_{2}=\cdots=n_{k}-r_{k}=s$ hold. Then the graph $G=G_{1} \nabla G_{2} \nabla \cdots \nabla G_{k}$ has $n=n_{1}+n_{2}+\cdots+n_{k}$ vertices and is regular of degree $r=n-s$, the characteristic polynomial of the join $G$ is given by

$$
\phi(G, x)=(x-r)(x+n-r)^{k-1} \prod_{i=1}^{k} \frac{\phi\left(G_{i}, x\right)}{x-r_{i}} .
$$

By Lemma 2.5, we have following Corollary.

Corollary 2.6. Let $G_{1}, G_{2}, \cdots, G_{k}$ be regular graphs, let $G_{i}$ have degree $r_{i}$ and $n_{i}$ vertices $(i=1,2, \cdots, k)$. where the relations $n_{1}-r_{1}=n_{2}-r_{2}=\cdots=n_{k}-r_{k}=s$ hold. Then

$$
\varepsilon\left(G_{1} \nabla G_{2} \nabla \cdots \nabla G_{k}\right)=2(k-1) s+\sum_{i=1}^{k} \varepsilon\left(G_{i}\right) .
$$

\section{The Unary Operations of Graphs}

Let $G$ be a graph with vertex set $V(G)=\left\{v_{1}, v_{2}, \cdots, v_{n}\right\}$, the duplication graph 
$D_{m} G$ is the graph with $m n$ vertices obtained from $m G$ by joining $v_{i}$ to each neighbors of $v_{i}$ in the $j$-th copy of $G(j=1,2, \cdots, m, i=1,2, \cdots, n)$.

Theorem 3.1. Let $G$ be a graph. Then

$$
\varepsilon\left(D_{m} G\right)=m \varepsilon(G) .
$$

Proof. If $A(G)$ is the adjacency matrix of graph $G$, then, it is obviously that the adjacency matrix of the duplication graph $D_{m} G$ is $J_{m} \otimes A(G)$, where $J_{m}$ is all 1 matrix of order $m$. the spectrum of $J_{m}$ is $m, 0(m-1)$ times, similar to the proof of Theorem 2.1, we have $\varepsilon\left(D_{m} G\right)=m \varepsilon(G)$.

Corollary 3.1. Let $G$ and $H$ be two equienergetic graph, then $D_{m} G$ and $D_{m} H$ are equienergetic.

Let $G$ be a graph, the line graph $L(G)$ of graph $G$ is the graph whose vertices are the edges of $G$, with two vertices in $L(G)$ adjacent whenever the corresponding edge in $G$ have exactly one vertex in common.

Lemma 3.1 [1] If $G$ is a regular graph of degree $r$, with $n$ vertices and $m\left(=\frac{1}{2} n r\right)$ edges, then

$$
\phi(L(G), x)=(x+2)^{m-n} \phi(G, x-r+2) .
$$

Corollary 3.2. Let $G$ be a regular graph of degree $r$, with $n$ vertices and $m\left(=\frac{1}{2} n r\right)$ edges, If $\lambda_{1}(=r), \lambda_{2}, \cdots, \lambda_{n}$ is the eigenvalues of $G$, then

$$
\varepsilon(L(G))=2(m-n)+\sum_{i=1}^{n}\left|r+\lambda_{i}-2\right|
$$

Corollary 3.3.

$$
\varepsilon\left(L\left(K_{n}\right)\right)= \begin{cases}2 n^{2}-6 n & 4 \leq n, \\ 4(n-2) & 2 \leq n \leq 3 .\end{cases}
$$

Let $G$ be a graph, the subdivision graph $S(G)$ of graph $G$ is the graph obtained by inserting a new vertex into every edge of $G$. The graph $R(G)$ of graph $G$ is the graph obtained from $G$ by adding, for each edge $u v$, a new vertex whose neighbours are $u$ and $v$. The graph $Q(G)$ of graph $G$ is the graph obtained from $G$ by inserting a new vertex into every edge of $G$, and joining by edges those pairs of new vertices which lie on adjacent edges of $G$. The total graph $T(G)$ of graph $G$ is the graph whose vertices are the vertices and edges of $G$, with two vertices of $T(G)$ adjacent if and only if the corresponding element of $G$ are adjacent or incident.

Lemma 3.2. [1] If $G$ is a regular graph of degree $r$, with $n$ vertices and $m\left(=\frac{1}{2} n r\right)$ edges, then

1) $\phi(S(G), x)=x^{m-n} \phi\left(G, x^{2}-r\right)$,

2) $\phi(R(G), x)=x^{m-n}(x+1)^{n} \phi\left(G, \frac{x^{2}-r}{x+1}\right)$,

3) $\phi(Q(G), x)=(x+2)^{m-n}(x+1)^{n} \phi\left(G, \frac{x^{2}-(r-2) x-r}{x+1}\right)$. 
4) The total graph $T(G)$ has $m-n$ eigenvalues equal to -2 and the following $2 n$ eigenvalues:

$$
\frac{1}{2}\left(2 \lambda_{i}+r-2 \pm \sqrt{4 \lambda_{i}+r^{2}+4}\right),(i=1,2, \cdots, n) .
$$

Theorem 3.2. Let $G$ be a regular graph of degree $r$, with $n$ vertices and $m\left(=\frac{1}{2} n r\right)$ edges, If $\lambda_{1}(=r), \lambda_{2}, \cdots, \lambda_{n}$ is the eigenvalues of $G$, then

1) $\varepsilon(S(G))=2 \sum_{i=1}^{n} \sqrt{r+\lambda_{i}}$,

2) $\varepsilon(R(G))=\sum_{i=1}^{n} \sqrt{\lambda_{i}^{2}+4\left(r+\lambda_{i}\right)}$,

3) $\varepsilon(Q(G))=2(m-n)+\sum_{i=1}^{n} \sqrt{\left(r+\lambda_{i}\right)^{2}+4}$,

4)

$$
\begin{aligned}
\varepsilon(T(G))= & 2(m-n)+\frac{1}{2} \sum_{i=1}^{n}\left(\left|2 \lambda_{i}+r-2+\sqrt{4 \lambda_{i}+r^{2}+4}\right|\right. \\
& \left.+\left|2 \lambda_{i}+r-2-\sqrt{4 \lambda_{i}+r^{2}+4}\right|\right) .
\end{aligned}
$$

Proof. (1) By Lemma 3.2 (1), we know that the spectrum of $S(G)$ is $\left\{0(m-n\right.$ times $\left.), \pm \sqrt{r+\lambda_{i}}(i=1,2, \cdots, n)\right\}$. So $\varepsilon(S(G))=2 \sum_{i=1}^{n} \sqrt{r+\lambda_{i}}$.

(2) By Lemma $3.2(2)$, we know that the spectrum of $R(G)$ is

$$
\begin{aligned}
& \left\{0(m-n \text { times }), \frac{\lambda_{i} \pm \sqrt{\lambda_{i}^{2}+4\left(r+\lambda_{i}\right)}}{2}(i=1,2, \cdots, n)\right\} . \text { So } \\
& \varepsilon(R(G))=\sum_{i=1}^{n} \sqrt{\lambda_{i}^{2}+4\left(r+\lambda_{i}\right)} .
\end{aligned}
$$

(3), (4) Proof is similar to (1).

Corollary 3.4. 1) If $n \geq 2$, then $\varepsilon\left(S\left(K_{n}\right)\right)=2(\sqrt{2 n-2}+(n-1) \sqrt{n-2})$.

2) If $n \geq 2$, then $\varepsilon\left(R\left(K_{n}\right)\right)=\sqrt{n^{2}+6 n-7}+(n-1) \sqrt{4 n-7}$.

3) $\varepsilon\left(Q\left(K_{n}\right)\right)=n^{2}-3 n+2 \sqrt{n^{2}-2 n+2}+(n-1) \sqrt{n^{2}-4 n+8}$.

4) If $n \geq 2$, then $\varepsilon\left(T\left(K_{n}\right)\right)= \begin{cases}2 n^{2}-2 n-4 & n \geq 3 \\ 4 & n=2 .\end{cases}$

\section{Conclusion}

In this paper, we prove that $\varepsilon(G \times H)=\varepsilon(G) \times \varepsilon(H), \quad \varepsilon\left(D_{m} G\right)=m \varepsilon(G)$. For regular graphs $G$ and $H$, we give the computational formulas of $\varepsilon(G \nabla H)$, $\varepsilon(G \circ H), \varepsilon(L(G)), \varepsilon(S(G)), \varepsilon(R(G)), \varepsilon(Q(G))$, and $\varepsilon(T(G))$ respectively. In particularly, we obtained a lot of graphs pair of equienergetic.

\section{Acknowledgements}

This work is supported by National Natural Science Foundation of China (11561056, 11661066), National Natural Science Foundation of Qinghai Provence (2016-ZJ-914), and Scientific Research Fund of Qinghai University for Nationalities (2015G02).

\section{References}

[1] Cvetković, D., Rowlinson, P. and Simić, S. (2010) An Introduction to the Theory of Graph Spectra. Cambridge University Press, Cambridge. 
[2] Gutman, I. (1978) The Energy of a Graph. Ber. Math.-Statist. Sekt. Forschungsz. Graz, 103, 1-22.

[3] Cvetković, D. and Gutman, I. (Eds.) (2009) Applications of Graph Spectra. Mathematical Institution, Belgrade.

[4] Graovac, A., Gutman, I. and Trinajstić, N. (1977) Topological Approach to the Chemistry of Conjugated Molecules. Springer, Berlin. https://doi.org/10.1007/978-3-642-93069-0

[5] Gutman, I. and Polansky, O.E. (1986) Mathematical Concepts in Organic Chemistry. Springer, Berlin. https://doi.org/10.1007/978-3-642-70982-1

[6] Gutman, I. and Trinajstić, N. (1973) Graph Theory and Molecular Orbitals. Topics Curr. Chem., 42, 49-93.

[7] Li, X.L., Shi, Y.T. and Gutman, I. (2012) Graph Energy. Springer, Berlin. https://doi.org/10.1007/978-1-4614-4220-2

[8] Balakrishnan, R. (2004) The Energy of a Graph. Linear Algebra and Its Applications, 387, 287-295.

[9] Ramane, H.S. and Walikar, H.B. (2007) Construction of Eqienergetic Graphs. MATCH Communications in Mathematical and in Computer Chemistry, 57, 203210.

[10] Bondy, J.A. and Murty, U.S.R. (1976) Graph Theory with Applications. MacMllan, London.

[11] Marcus, M. and Minc, H. (1964) A Survey of Matrix Theory and Matrix Inequalities. Allyn and Bacon. Inc., Boston.

Submit or recommend next manuscript to SCIRP and we will provide best service for you:

Accepting pre-submission inquiries through Email, Facebook, LinkedIn, Twitter, etc. A wide selection of journals (inclusive of 9 subjects, more than 200 journals)

Providing 24-hour high-quality service

User-friendly online submission system

Fair and swift peer-review system

Efficient typesetting and proofreading procedure

Display of the result of downloads and visits, as well as the number of cited articles

Maximum dissemination of your research work

Submit your manuscript at: http://papersubmission.scirp.org/

Or contact apm@scirp.org 